\title{
DNA methylation and gene expression integration in cardiovascular disease
}

\author{
Guillermo Palou-Márquez ${ }^{1,2,3}$, Isaac Subirana ${ }^{1,4}$, Lara Nonell ${ }^{5}$, Alba Fernández-Sanlés ${ }^{1,6 \dagger}$ and \\ Roberto Elosua ${ }^{1,7,8^{*}+}$ (D)
}

\begin{abstract}
Background: The integration of different layers of omics information is an opportunity to tackle the complexity of cardiovascular diseases (CVD) and to identify new predictive biomarkers and potential therapeutic targets. Our aim was to integrate DNA methylation and gene expression data in an effort to identify biomarkers related to cardiovascular disease risk in a community-based population. We accessed data from the Framingham Offspring Study, a cohort study with data on DNA methylation (Infinium HumanMethylation450 BeadChip; Illumina) and gene expression (Human Exon 1.0 ST Array; Affymetrix). Using the MOFA2 R package, we integrated these data to identify biomarkers related to the risk of presenting a cardiovascular event.

Results: Four independent latent factors (9, 19,21 —only in women —and 27), driven by DNA methylation, were associated with cardiovascular disease independently of classical risk factors and cell-type counts. In a sensitivity analysis, we also identified factor 21 as associated with CVD in women. Factors 9, 21 and 27 were also associated with coronary heart disease risk. Moreover, in a replication effort in an independent study three of the genes included in factor 27 were also present in a factor identified to be associated with myocardial infarction (CDC42BPB, MAN2A2 and RPTOR). Factor 9 was related to age and cell-type proportions; factor 19 was related to age and B cells count; factor 21 pointed to human immunodeficiency virus infection-related pathways and inflammation; and factor 27 was related to lifestyle factors such as alcohol consumption, smoking and body mass index. Inclusion of factor 21 (only in women) improved the discriminative and reclassification capacity of the Framingham classical risk function and factor 27 improved its discrimination.

Conclusions: Unsupervised multi-omics data integration methods have the potential to provide insights into the pathogenesis of cardiovascular diseases. We identified four independent factors (one only in women) pointing to inflammation, endothelium homeostasis, visceral fat, cardiac remodeling and lifestyles as key players in the determination of cardiovascular risk. Moreover, two of these factors improved the predictive capacity of a classical risk function.
\end{abstract}

Keywords: DNA methylation, Gene expression, Multi-omics integration, Cardiovascular disease, MOFA, Unsupervised integration

\footnotetext{
*Correspondence: relosua@imim.es

${ }^{\dagger}$ Alba Fernández-Sanlés and Roberto Elosua have equally contributed to this work

${ }^{1}$ Cardiovascular Epidemiology and Genetics Research Group, Hospital del Mar Medical Research Institute (IMIM), Dr Aiguader 88, 08003 Barcelona, Spain

Full list of author information is available at the end of the article
}

\section{Background}

Cardiovascular diseases (CVD) are the leading cause of mortality and disease burden worldwide $[1,2]$ and comprise several diseases with different etiologies that affect the heart or blood vessels. CVD prevention, one of the main public health challenges, is based on population and individual interventions [3]. The 
former includes strategies affecting the whole population, such as smoking ban policies, whereas individual interventions are tailored to each patient based on the estimation of cardiovascular risk. Cardiovascular risk functions are the most common tool to assess cardiovascular risk. Several functions have been developed and validated; however, their sensitivity is low, as a significant number of CVD events occur in individuals with a low or moderate 10-year risk [4]. Therefore, it is necessary to identify and evaluate new predictive biomarkers to improve cardiovascular risk estimation. Moreover, despite pharmacological success in reducing cardiovascular morbidity and mortality, the search for new pathogenic pathways and therapeutic targets is important because residual cardiovascular risk remains a major concern [5].

CVD comprises complex heterogeneous diseases, resulting from an interplay between omic, physiological, environmental and lifestyle factors. Atherosclerosis is the main common pathogenic mechanism, and individual omic analyses have identified markers associated with atherosclerotic CVD. For instance, genome-wide association studies have identified more than 150 loci related to coronary heart disease (CHD) [6], and epigenome-wide association studies (EWAS) have identified several CpGs showing differential methylation related to CVD risk [7-9]. DNA methylation is one of the mechanisms regulating gene expression, which could also determine CVD risk [10]. However, none of the omic layers of biological information (e.g., genomic, epigenomic, transcriptomic, proteomic, metabolomic) captures the full complexity of CVD.

The integration of different layers of omics information is an opportunity to tackle the complexity of CVD and to identify new predictive biomarkers and potential therapeutic targets [11]. Although this integrative analysis remains challenging because of inherent data-type differences, the field is growing and several methods have already been implemented [12]. These methods can be classified as supervised and unsupervised. The aim of supervised methods is to predict one or more conditions related to a sample, although overfitting may be a concern. In contrast, unsupervised methods explore the data by analyzing the correlations among samples in order to condense or simplify the large volume of data in a reduced number of factors that in turn could be associated with clinical traits. One of these unsupervised methods is multi-omics factor analysis (MOFA) [13, 14].

The aim of this study was to integrate DNA methylation and gene expression data to identify biomarkers related to the risk of presenting a cardiovascular event in the Framingham Offspring Study (FOS) using an unsupervised method.

\section{Results}

Quality control of DNA methylation and gene expression datasets

From 485,577 CpGs and 2620 samples, 411,019 CpGs and 2055 samples remained after the quality control of the DNA methylation data and the application of inclusion and exclusion criteria (Additional file 2: Fig. S1). From 22,011 transcripts and 1,200 samples, 19,904 transcripts and 914 samples were considered for analysis after the quality control of the gene expression data and the application of inclusion and exclusion criteria (Additional file 2: Fig. S2). In this process, we removed all individuals from the transcriptomic batch 15 in both omic datasets (24 samples in transcriptomics and 25 samples in DNA methylation), as this batch showed a differentiated clustering pattern from the rest of the samples.

The main sociodemographic and clinical characteristics of the analyzed individuals are shown in Table 1. Their characteristics were similar to individuals not included in the analysis.

\section{Identification of MOFA factors related to CVD using an omics integration approach: main analysis}

We used the MOFA2 $R$ package to integrate the omics data and identify factors related to the CVD. The 30 identified factors explained $83.35 \%$ of the variance of both omics, $45.48 \%$ explained by gene expression and $37.87 \%$ by DNA methylation (Fig. 1). Surprisingly, most of the

Table 1 Descriptive characteristics of the Framingham Offspring Study participants included in this integration analysis

\begin{tabular}{|c|c|c|}
\hline Variable & $\begin{array}{l}\text { Methylation } \\
n=2055\end{array}$ & $\begin{array}{l}\text { Gene expression } \\
n=914\end{array}$ \\
\hline $\mathrm{Age}^{\mathrm{a}}$ & $65.23(8.59)$ & $64.48(8.43)$ \\
\hline Sex, male, $n(\%)$ & $871(42.38)$ & $336(36.76)$ \\
\hline Total cholesterol, mg/dL ${ }^{a}$ & $190.16(35.79)$ & $192.30(35.62)$ \\
\hline $\mathrm{HDL}-\mathrm{C}, \mathrm{mg} / \mathrm{dL}^{\mathrm{a}, \mathrm{c}}$ & $58.91(18.45)$ & $59.51(17.96)$ \\
\hline Triglycerides, mg/dL b,c & $100(73,138)$ & $101(73,140.75)$ \\
\hline $\mathrm{SBP}, \mathrm{mmHg}{ }^{\mathrm{a}, \mathrm{c}}$ & $125.19(16.83)$ & 125.35 (16.97) \\
\hline $\mathrm{DBP}, \mathrm{mmHg} \mathrm{g}^{\mathrm{a}, \mathrm{c}}$ & $72.31(9.90)$ & $72.65(10.16)$ \\
\hline Glucose, mg/dL ${ }^{b}$ & $101(94,109)$ & $100(93,108)$ \\
\hline Smokers, $n(\%)$ & 199 (9.68) & $96(10.50)$ \\
\hline $\mathrm{BMl}, \mathrm{kg} / \mathrm{m}^{2 \mathrm{a}, \mathrm{c}}$ & $27.99(5.30)$ & $27.88(5.33)$ \\
\hline Waist, $\mathrm{cm}^{\mathrm{a}, \mathrm{c}}$ & $100.56(14.47)$ & $100.08(14.67)$ \\
\hline $\mathrm{CHD}, n(\%)$ & $83(4.04)$ & $28(3.06)$ \\
\hline CVD, $n(\%)$ & $201(9.78)$ & 79 (8.64) \\
\hline
\end{tabular}

$S B P$ systolic blood pressure, $D B P$ diastolic blood pressure, $B M I$ body mass index, Waist waist circumference, $C H D$ coronary heart disease, $C V D$ cardiovascular disease

${ }^{\text {a }}$ Mean (standard deviation)

b Median (interquartile range)

c HDL-C, high-density lipoprotein cholesterol 


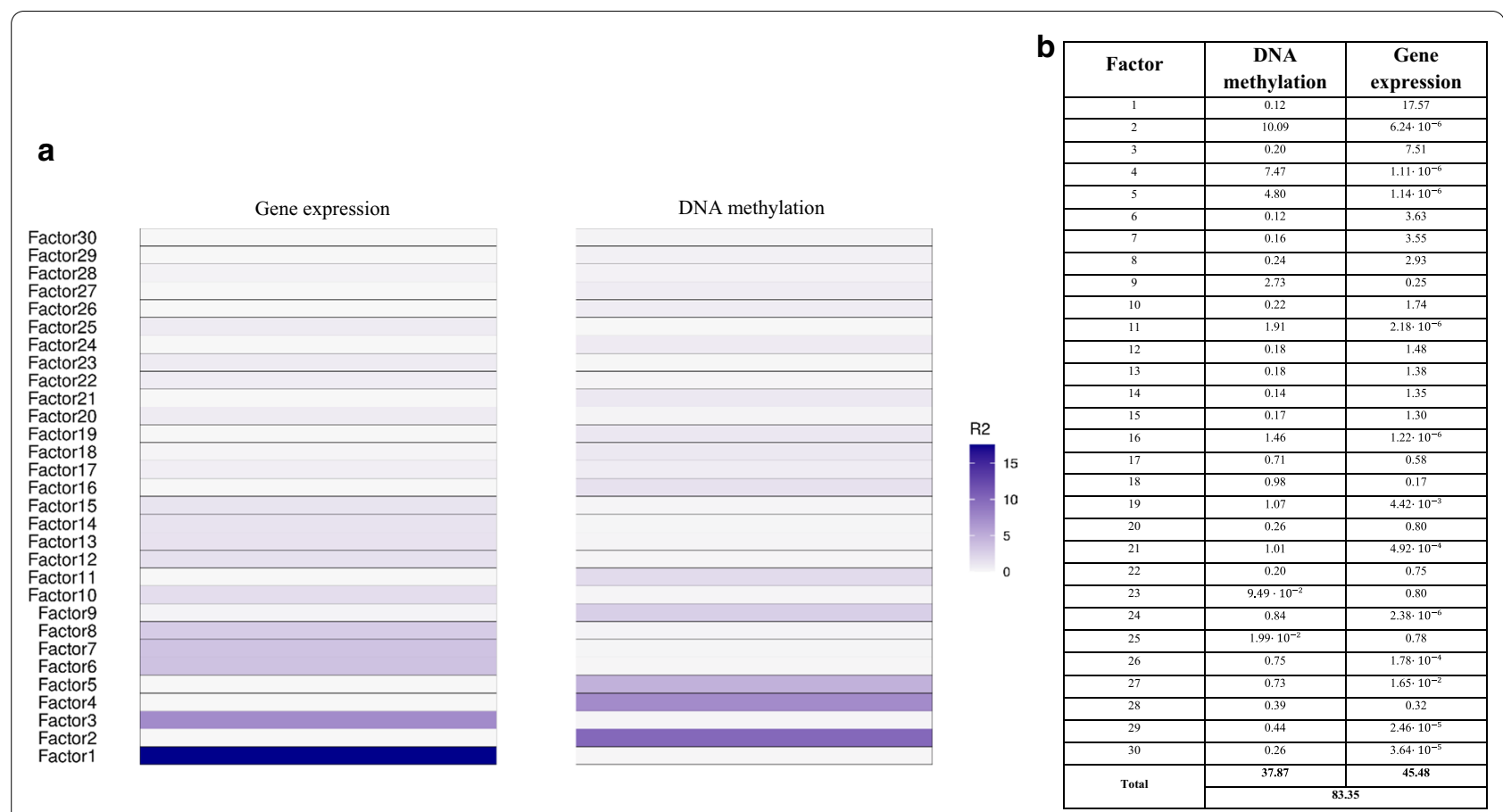

Fig. 1 Variance $\left(R^{2}\right)$ explained by each omic in each factor. a Variance explained in a blue-tone color scale. b Absolute percentage values of variance explained by each omic in each factor and the total variance explained by all 30 factors

factors were mainly explained by only one of the two integrated omics. Correlation coefficients among factors were $<0.20$ (Additional file 2: Fig. S3).

\section{Association between the identified MOFA factors and CVD incidence}

The median follow-up of the population was 7.7 years. We first assessed the correlations between the 30 MOFA factors, the main covariates and CVD incidence (Fig. 2). The 30 MOFA factor violin plots stratified by CVD are shown in Additional file 2: Fig. S4. In the main univariate analysis, four factors $[9,19,21,27]$ were associated with CVD risk (Table 2 and Fig. 3). These factors were mostly driven by DNA methylation (Fig. 1). The associations between the four factors and covariates are shown in Additional file 1: Table S1. Factor 9 was mainly related to age, CD4 + T, CD8 + T and NK cells; factor 19 to age and B cells; factor 21 to sex; and factor 27 to B cells.

In the main multivariate analyses, factors 9, 19 and 27 were associated with CVD independently of classical risk factors. We also found an interaction between factor 21 and sex on CVD risk ( $p$-value $=0.007$ on model 3 ); therefore, the analyses were additionally stratified by sex. This factor was associated with CVD only in women.

As expected, most of the CpGs included in the analyses had weight values close to zero in the factors 9, 19, 21 and 27, whereas a few CpGs showed large absolute values, indicating a strong association with the factors (Additional file 2: Fig. S5). We identified the $30 \mathrm{CpGs}$ with the highest weights in those factors (Additional file 2: Fig. S6). The correlation coefficients among the CpGs for each factor are shown in Additional file 2: Fig. S7.

Out of the selected $30 \mathrm{CpGs}$ of each factor, 29, 14, 17 and 13 CpGs of factors 9, 19, 21 and 27, respectively, showed an association with CVD (nominal FDR $p$-value $<0.01$, Additional file 1: Tables S2-S5) in the multivariate analysis adjusted for cell-type proportions and one surrogate variable.

\section{Evaluation of the clinical relevance of the CVD-related factors}

We then evaluated the predictive value of including the significant factors in the Framingham risk function (Table 2). The inclusion of factors 21 (only in women) and 27 improved the capacity to discriminate CVD events in the FOS cohort. Reclassification improvement was observed for factor 21 in women, both in the whole group of women and in those with intermediate risk (clinical reclassification).

\section{Sensitivity analyses and replication of the top features from the CVD-related factors in an independent study} We performed a sensitivity analysis in which we selected the 20,000 CpGs showing the highest 


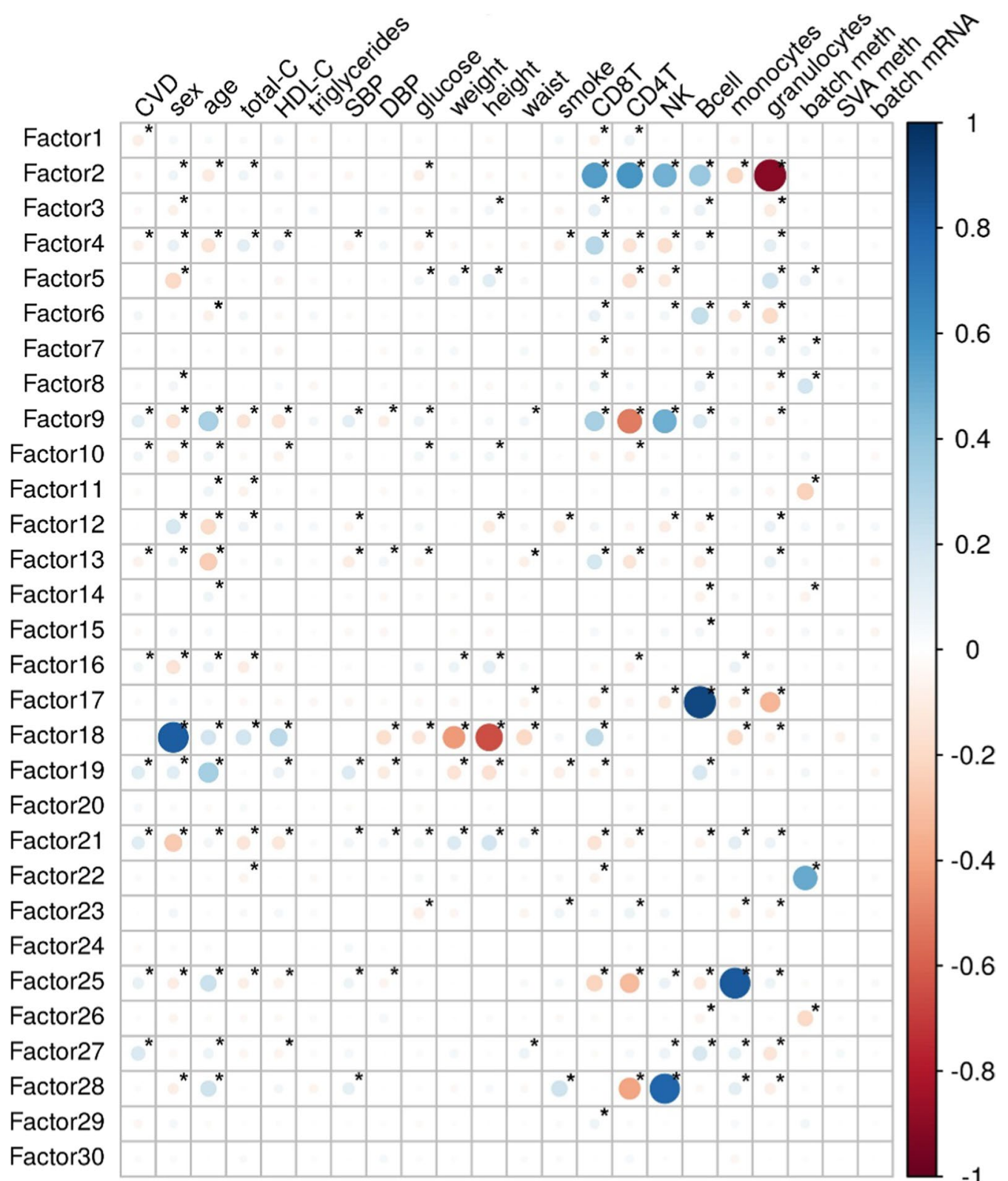

Fig. 2 Correlation between the MOFA factors, cardiovascular disease (CVD) incidence and the covariates. Correlation coefficients are represented in a color scale from red, for negative correlations, to blue, for positive correlations. *Statistically significant correlation coefficients

variability instead of the most significantly associated with CVD (main analyses). MOFA identified one factor independently associated with CVD. This factor was similar to factor 21 from the main analyses and included CpGs associated with HIV infection pathways, as well as cg06642177, which has been previously related to myocardial infarction.

As a different sensitivity analysis, we also assessed the association of the four identified factors with CHD and found that factors 9, 21 (in women) and 27 showed a similar effect size of association with the two outcomes (Additional file 1: Table S6).

The independent replication was conducted in a casecontrol study of 391 individuals of the REGICOR -REgistre GIroní del COR-study (196 cases and 195 controls), in which 811,610 CpGs were available after the quality control. In this study, we identified 30 MOFA factors and 10 were associated with myocardial infarction; one of them included three genes that were also included in the factor 27 of the FOS cohort: $C D C 42 B P B, M A N 2 A 2$, and 
Table 2 Association of the MOFA factors and cardiovascular disease risk (Cox regression): Model 1, adjusted for cell-type counts and one surrogate variable; Model 2, additionally adjusted for age and sex; Model 3, additionally adjusted for total cholesterol, HDL-C levels, glucose, smoking status and systolic and diastolic blood pressure

\begin{tabular}{|c|c|c|c|c|c|c|c|c|}
\hline \multirow[t]{2}{*}{ Model } & \multicolumn{3}{|c|}{ Association with CVD incidence } & \multicolumn{5}{|c|}{ Predictive capacity of the Framingham CVR function } \\
\hline & $\mathrm{HR}^{\mathrm{a}}\left(95 \% \mathrm{Cl}^{\mathrm{a}}\right)$ & $p$-value & FDR correction & $\begin{array}{l}\text { C-statistic } \\
\text { classical } \\
\text { function }\end{array}$ & $\begin{array}{l}\text { C-statistic } \\
\text { classical } \\
\text { function + factor }\end{array}$ & $p$-value ${ }_{C}^{a}$ & $\left.N R\right|^{a}(95 \% \mathrm{Cl})$ & $\begin{array}{l}\text { Clinical NRI }{ }^{a}(95 \% \\
\left.C^{a}\right)\end{array}$ \\
\hline F9a - Model 1 & $2.05(1.69,2.48)$ & $3.04 \times 10^{-13}$ & $9.44 \times 10^{-12}$ & - & - & - & - & - \\
\hline F9-Model 2 & $1.56(1.26,1.93)$ & $3.48 \times 10^{-5}$ & $2.7 \times 10^{-4}$ & - & - & - & - & - \\
\hline F9-Model 3 & $1.42(1.15,1.77)$ & $1.37 \times 10^{-3}$ & $8.46 \times 10^{-3}$ & 0.73 & 0.73 & 0.97 & $\begin{array}{l}-2.12(-8.17 \\
3.89)\end{array}$ & $\begin{array}{l}-2.38(-10.74 \\
5.98)\end{array}$ \\
\hline F19 & $1.42(1.26,1.61)$ & $9.10 \times 10^{-9}$ & $7.05 \times 10^{-8}$ & - & - & - & - & - \\
\hline F19-Model 2 & $1.21(1.06,1.38)$ & $4.61 \times 10^{-3}$ & $2.38 \times 10^{-2}$ & - & - & - & - & - \\
\hline F19-Model 3 & $1.20(1.05,1.37)$ & $9 \times 10^{-3}$ & $4.65 \times 10^{-2}$ & 0.73 & 0.74 & 0.20 & $\begin{array}{l}0.21(-8.01 \\
8.01)\end{array}$ & $1.85(-8.83,12.54)$ \\
\hline $\begin{array}{l}\mathrm{F} 21^{\mathrm{a}} \mathrm{M}^{\mathrm{a}} \text {-Model } \\
\end{array}$ & $1.24(1.02,1.51)$ & $3.22 \times 10^{-2}$ & $6.65 \times 10^{-2}$ & - & - & - & - & - \\
\hline F21 M-Model 2 & $1.21(1.00,1.48)$ & $5.56 \times 10^{-2}$ & 0.13 & - & - & - & - & - \\
\hline F21 M-Model 3 & $1.21(0.99,1.48)$ & $6.38 \times 10^{-2}$ & 0.15 & 0.71 & 0.72 & 0.30 & $\begin{array}{l}2.92(-8.03 \\
13.49)\end{array}$ & $6.61(-9.53,22.76)$ \\
\hline $\begin{array}{c}\text { F21 Wa_-Model } \\
1\end{array}$ & $1.81(1.44,2.29)$ & $5.52 \times 10^{-7}$ & $3.42 \times 10^{-6}$ & - & - & - & - & - \\
\hline F21 W-Model 2 & $1.71(1.36,2.15)$ & $5.54 \times 10^{-6}$ & $5.72 \times 10^{-5}$ & - & - & - & - & - \\
\hline F21 W-Model 3 & $1.77(1.39,2.24)$ & $2.40 \times 10^{-6}$ & $3.72 \times 10^{-5}$ & 0.75 & 0.79 & 0.01 & $\begin{array}{c}20.85(5.04 \\
37.38)\end{array}$ & $24.00(4.55,43.43)$ \\
\hline F27 $7^{\mathrm{a}}$-Model 1 & $1.38(1.25,1.53)$ & $5.98 \times 10^{-10}$ & $9.28 \times 10^{-9}$ & - & - & - & - & - \\
\hline F27-Model 2 & $1.38(1.25,1.54)$ & $4.48 \times 10^{-10}$ & $1.39 \times 10^{-8}$ & - & - & - & - & - \\
\hline F27-Model 3 & $1.36(1.22,1.51)$ & $1.08 \times 10^{-8}$ & $3.35 \times 10^{-7}$ & 0.73 & 0.75 & 0.01 & $\begin{array}{l}1.23(-6.48 \\
8.44)\end{array}$ & $4.90(-4.37,14.17)$ \\
\hline
\end{tabular}

Cell-type counts and one surrogate variable were used as covariates in the three models. Factor 21 was stratified by sex, as the interaction between this factor and sex was statistically significant. The predictive added-value of each factor when included in the Framingham risk function is also shown in terms of discrimination improvement (C-statistic) and reclassification (Net Reclassification Improvement)

a CVD, cardiovascular disease; $\mathrm{HR}$, hazard ratio; $\mathrm{Cl}$, confidence interval; $p$-value ${ }_{\mathrm{C}}$, $p$-value of the c-statistic comparison; NRI, net reclassification improvement; $\mathrm{F9}$, factor 9; F19, factor 19; F21, factor 21; F27, factor 27; M, men; W, women

RPTOR (Additional file 1: Table S5). None of the top 30 CpGs from factors 9, 19, 21 and 27 were replicated in the REGICOR population (Additional file 1: Tables S7-S10).

\section{Discussion}

We used an unsupervised machine-learning method (MOFA) to identify latent factors that capture biological and technical sources of variability in DNA methylation and gene expression datasets. By integrating these omic data, we identified three factors, almost exclusively explained by DNA methylation, that were independently associated with CVD: factor 19, which included CpGs previously related to age; factor 21 (only in women), which included CpGs previously related to HIV infection pathways and myocardial infarction; and factor 27, which included CpGs previously related to lifestyle factors. Moreover, we report that the inclusion of factor 21 (in women) and factor 27 in the classical Framingham risk function improved its predictive capacity by increasing the discrimination or reclassification.

\section{Omics integration}

The integration of several omics allows modeling data to disentangle the molecular architecture and biological processes of complex traits. Several methods have been proposed for the integration of omic data [15], including MOFA. This method has several advantages, such as identifying latent factors that explain the variability across one or several types of omic data, and the inclusion of samples with missing data in one of the analyzed omic datasets. Among its limitations, as an unsupervised method, are its use of exploratory data analysis to generate hypotheses, the challenge of achieving consistent results and overfitting of the results, although the results seem to be robust in large samples [16].

The added value of data integration was not clearly evidenced in this study, as the identified factors associated 


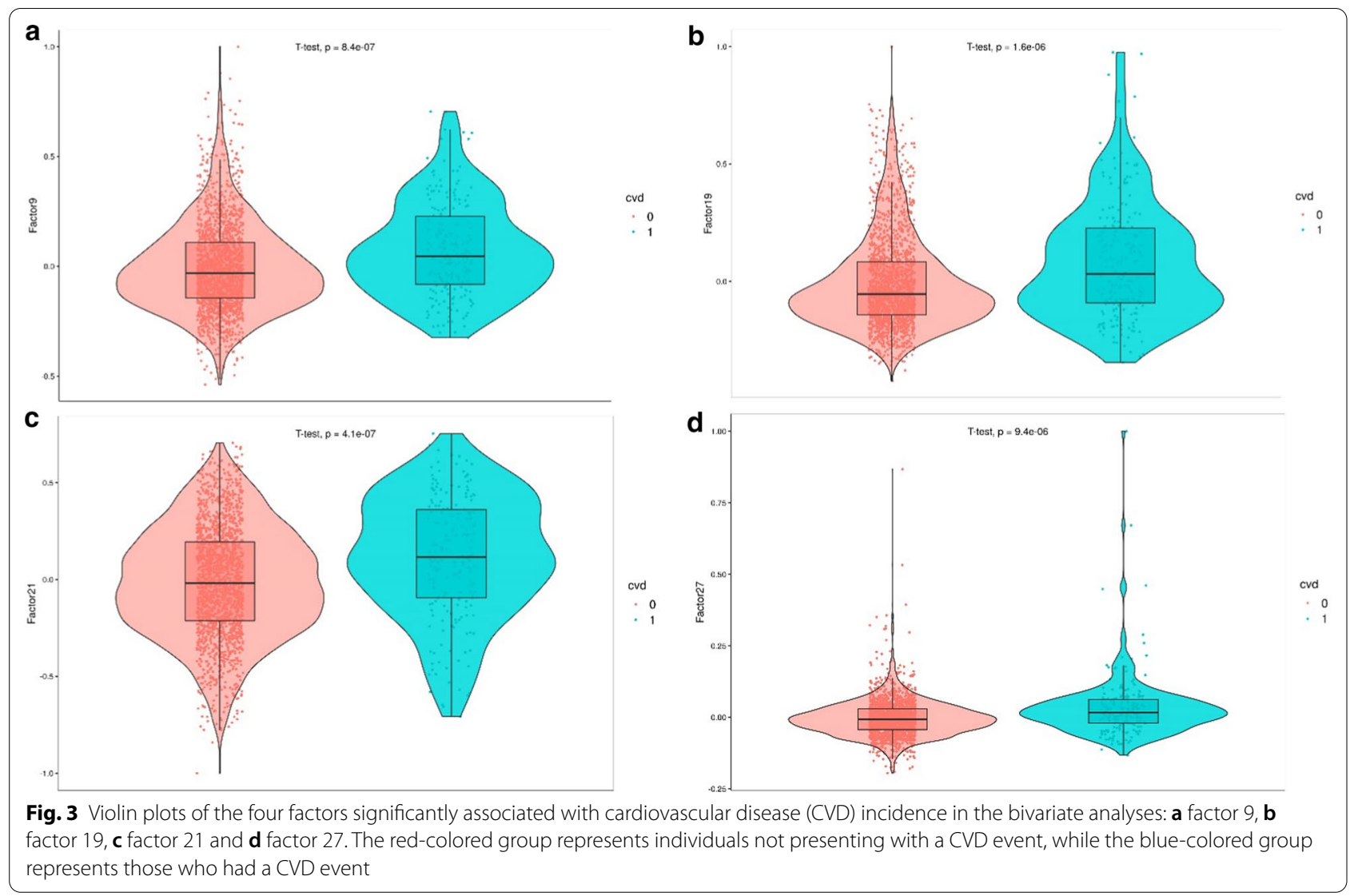

with CVD were almost exclusively driven by DNA methylation. However, MOFA is also useful to detect features related to a single omic and latent factors can give more insights into the etiology of CVD, as they offer an integrated understanding and synthesis of the CVD-related molecular pathways and incorporates complex interrelationships across CpGs. This approach could prove to be more useful than the analysis of individual methylation markers.

We aimed to homogenize the number of epigenome and transcriptome data points to be included in the MOFA analysis. As gene expression data included 22,011 transcripts and all of them were included in the main MOFA2 analysis, we selected methylation data to include $20,000 \mathrm{CpGs}$ of the original 411,019. Two main strategies could be used to select 20,000 CpG: either select them based on their variability or based on their association with the outcome of interest (CVD). We selected the latter to enrich our initial dataset with marks showing association with CVD. However, this approach enriches methylation data but not transcriptomic data, and it could explain why the factors associated with CVD only included DNA methylation attributes. Therefore, we conducted a sensitivity analysis based on the $\mathrm{CpG}$ variability selection criteria, which identified one MOFA factor independently associated with CVD. This factor only included DNA methylation attributes and was similar to factor 21 .

\section{Identified molecular markers: biological pathways}

In this study, we identified four factors related to CVD: 9, 19, 21 (in women) and 27. In a sensitivity analysis focusing on CHD, we found that three of the identified factors were also related to CHD with similar effect sizes to those found in the main analysis with CVD: factors 9, 21 (in women) and 27. Factor 19 was not related to CHD but its association with CVD was marginally significant $(\mathrm{HR}=1.20, \mathrm{FDR} p$-value $=0.047)$. The consistency between analyses points to atherosclerosis-related pathways.

MOFA, as an unsupervised method, only considers methylation and transcriptomic variability, so the identification of the latent factors does not account for covariates. Therefore, some latent factors could reflect variability in cell-type counts in blood, without changes in the molecular characteristics in any of the mature cells of the blood. This phenomena is called polycreodism [17], which in this study is particularly important 
to account for since cell-type differences could reflect immune-related inflammation, a well-known pathogenic mechanism of atherosclerosis. Thus, the association between MOFA factors and CVD was adjusted for blood cell-type counts to mitigate their potential confounder effect.

Factor 9 was related to age and cell-type proportions. Some of the genes included in this factor have been previously related to cardioprotective effects: SLC1A5, SLP1 [18, 19]. However, the association with CVD was independent of age and cell types. Other genes clustered in this factor are GALNT2 that shows differential methylation associated with CHD [20], and PTP4A2 and JAZF1 that have been related to angiogenesis [21, 22].

Among the genes showing differential methylation features and included in factor 19, we can highlight $M C F 2 L, Z B T B 46$, ANGPTL2, and BICD2. Genetic variants in $M C F 2 L$ and $Z B T B 46$ have been reported to be significantly associated with CHD [23]. ANGPTL2 maintains vascular endothelium homeostasis, having a role in angiogenesis, tissue repair, obesity and atherosclerotic diseases [24]. Finally, genetic variants in BICD2 have been associated with visceral fat [25]. In summary, this factor suggests several biological factors (inflammation, endothelial homeostasis, visceral fat accumulation) that could explain the association with higher CVD risk.

Factor 21 was associated with CVD exclusively in women. Interestingly, this factor was also observed in the MOFA sensitivity analysis based on the CpG variability selection criteria. Moreover, 16 of the 30 top attributes included in factor 21 were also associated with CHD in the Framingham dataset in a previous integration effort using genomic and epigenomic data and a Random Forest classification model [26]. Twenty-nine out of $30 \mathrm{CpGs}$ from factor 21 have been associated with HIV infectionrelated pathways [27]. Among the genes showing differential methylation features and included in factor 21 , we can highlight NLRC4, NCL, PTEN, ATM, and SGK1. $N L R C 4$ and NCL contain genetic variants associated with inflammation biomarkers [28, 29]. Genetic variants in PTEN and ATM genes have been associated with eosinophil count [30] and CHD [31], respectively. Finally, differential methylation in cg06642177 linked to SKG1 has been previously associated with myocardial infarction [32]. This gene has been considered an important factor in the regulation of inflammation in CVD [33] and contributes to cardiac remodeling and development of heart failure [34]. In summary, this factor points to inflammation, cell cycle regulation and cardiac remodeling as key pathways in CVD risk. We do not have a clear explanation for the differential association with CVD between sexes.
Lastly, factor 27 was mainly related with lifestyle factors: alcohol consumption, body mass index and smoking. Interestingly, we replicated a similar factor including three common genes in an independent case-control study applying the MOFA analysis in REGICOR data. These genes (CDC42BPB, MAN2A2, RPTOR) present differential methylation related to alcohol consumption [35], body mass index [36] and smoking [37], respectively. Genetic variability in MAN2A2 and RPTOR has been related to CHD [38] and body mass index and blood pressure [39], respectively. Finally, another interesting gene included in factor 27 is $A B C A 2$ that reduces lowdensity lipoprotein receptor expression [40]. In summary, this factor suggests several biological mechanisms that could mediate the relationship between lifestyle factors and CVD risk.

Our analysis did not replicate previous findings from the Framingham heart study in which they reported, in combination with other cohorts, several CpGs or gene expression signatures related to myocardial infarction and CHD [8, 41]. However, our analysis approach using MOFA latent factors differs from those previously used and could explain these differences.

\section{Identified molecular markers: clinical predictive added-value}

Factors 21 (in women) and 27 improved the discriminative capacity of the Framingham risk function to identify individuals who will develop a CVD in the next 10 years. Reclassification improvement was significant in women for factor 21, as well as in the subgroup of women with intermediate risk. These reclassification results should be replicated in an independent prospective sample.

\section{Strengths and limitations}

The main strength of this study is the large sample size and the community-based design, along with its integrative approach to identify molecular markers related to CVD. In addition, the matrix factorization model of MOFA allows data treatment for individuals with missing values for one of the omics. We should consider the presence of population stratification and familiar relatedness and their potential effects in our results [42]. Potential population stratification would be accounted for using the MOFA latent factors (similar to methylation-based principal components) and surrogate variables, reducing the possibility of reporting false positive results [43]. However, we could not account for familiar relatedness in our analyses to minimize its potential impact on our results. Moreover, we are aware of additional limitations of the study: (1) the number of cases is limited, hampering the statistical power of the study; (2) not all the samples with transcriptomic data could be incorporated 
in the analysis because of a computational memory limitation; (3) the dimensions of the methylation data were reduced to match the dimensions of the available transcriptomics data, to avoid overrepresentation bias in the factors; (4) we did not replicate the complete analysis in an independent cohort as we did not have access to other populations with data of both omics; (5) MOFA modeling assumes linear association; thus, it does not consider nonlinear relationships between features within and across assays [44]; and (6) CVD include several clinical diseases, introducing some heterogeneity in our main outcome, although the main results for factors 9,21 and 27 are robust when analyzing CHD.

\section{Conclusions}

This study showed the potential of unsupervised integration methods to provide some insights in the pathogenesis of cardiovascular diseases. We identified four independent factors (one only in women) pointing to inflammation, endothelium homeostasis, visceral fat, cardiac remodeling and lifestyles as key players in the determination of cardiovascular risk. Two of these factors improved the predictive capacity of a classical risk function.

\section{Methods}

\section{Study design and population}

The Framingham Offspring Study (FOS) is a prospective community-based cohort study. FOS data were obtained through the database of Genotypes and Phenotypes (dbGAP, http://dbgap.ncbi.nlm.nih.gov; project number \#9047). We included the participants in exam 8 with available DNA methylation data (Framingham Offspring Exam 8 DNA Methylation Study, $n=2620$; dbGaP Study Accession: phs000724.v7.p11) and gene expression data (NHLBI Framingham SABRe CVD, $n=1892$; dbGaP Study Accession: phs000363.v17.p11). Participants with previous CVD and those with no follow-up data were excluded.

\section{DNA methylation assessment}

DNA extraction and methylation assessment have been previously fully described [45]. Briefly, DNA was extracted from buffy coat using a standardized method (Puregen TM, Gentra Systems). Genome-wide DNA methylation was assessed using the Infinium HumanMethylation450 BeadChip (Illumina, CA, USA), following the Illumina Infinium HD Methylation protocol [46, 47]. This array is based on the bisulfite conversion of 485,577 unmethylated cytosines across the genome.

The quality control protocol excluded cross-reactive probes $[48,49]$ and CpGs with a beadcount $<3$ in at least $5 \%$ of the samples and detection $p$-values $>0.05$ in at least $1 \%$ of the samples. We also excluded the samples with inconsistent methylation-based predicted and reported sex. Quality control was performed using the wateRmelon (v1.22.0) [50] and minfi (v1.24.0) [51] $\mathrm{R}$ packages. We also excluded CpGs located on the sexual chromosomes.

Methylation data were normalized using the Dasen method [50], which involves background adjustment of the methylated and unmethylated intensities, followed by between-array normalization and dye bias correction. The potential presence of batch effect was explored in a multi-dimensional scaling (MDS) plot, and if present it was controlled by regressing out the batch variable using ComBat [52].

Methylation status at each $\mathrm{CpG}$ site was reported by $M$-value. M-values above 4 standard deviations from the average in absolute value were excluded from analysis.

Finally, FlowSorted.Blood.450 k R package (v1.16.0) [53] was used to obtain methylation-based estimates of the blood cell-type counts (B Cells, Monocytes, Granulocytes, Natural Killers, CD8 + T cells and CD4+ T cells). The sva R package (v3.26.0) [54] was used to obtain surrogate variables to account for unmeasured technical or biological variability.

\section{Gene expression assessment}

RNA extraction and gene expression profiling have been previously described [41]. In brief, fasting peripheral whole blood samples were collected in PAXgene ${ }^{\mathrm{TM}}$ tubes (PreAnalytiX, Hombrechtikon, Switzerland). RNA was isolated and cDNA was obtained according to the manufacturer's standard protocols. cDNA was hybridized to the Human Exon 1.0 ST Array (Affymetrix, Inc., Santa Clara, CA). This array consists of over 6 million probes grouped in about 1.2 million probesets, targeted to the majority of known exons in the human genome. Only gene-level analysis (transcript clusters with "core" annotations) was conducted, including 22,011 transcripts.

Computational memory limited the analysis to 1,200 individuals, which we randomly selected from the available 1,892 . Quality control of the raw data was performed using the oligo R package (v1.42.0) [55]. We visualized the expression data for the analyzed samples, clustered by batch, using boxplots, Normalized Unscaled Standard Error (NUSE) and Relative Log Expression (RLE) plots. We considered as a potential outlier any sample whose median was above $95 \%$ or below $5 \%$ quantiles from the distribution of medians for each type of plot. A potential outlier observed in at least 2 out of 3 plots was considered a real outlier and removed from the data. Distribution of the red/green intensity ratio ('M') plotted by the average intensity ('A')-MA-plots-was also performed. Data were quantile-normalized, log2 transformed, 
background substracted and summarized by the Robust Multi-array Average method (RMA) [56] implemented in the oligo package. We removed transcripts with an expression value less than 4 in at least as many samples as the smallest experimental group (201 individuals with CVD). Finally, transcripts located on sexual chromosomes were removed. We explored for batch effect using MDS plots, and if present controlled for it by regressing out the batch variable with ComBat [52]. The group of participants with gene expression data was a subset of the DNA methylation set of participants.

\section{Clinical cardiovascular events and other covariates assessment}

The main clinical outcome was incident CVD that included coronary heart disease (angina, myocardial infarction, coronary revascularization and coronary heart disease death) and other cardiovascular events (heart failure, stroke, transient ischemic accident, carotid revascularization, peripheral artery disease and other circulatory problems). The events were adjudicated by the Framingham event committee. Follow-up included exam 8 (baseline visit) to exam 12. Traditional risk factors at the baseline visit (sex, age, total cholesterol, highdensity lipoprotein cholesterol [HDL-C], glucose, systolic and diastolic blood pressure [SBP and DBP, respectively] and smoking status) were used as covariates in the Cox regression analyses.

\section{MOFA models}

To perform the integration of both omics, we used the MOFA2 R package (v0.99.5) [13]. MOFA identifies latent factors that capture biological and technical sources of variability in multi-omics datasets. Mathematically, each factor orders cells through a one-dimensional axis centered at zero. The interpretation of factors is analogous to the interpretation of principal components.

The matrix of methylation data was much larger than the gene expression matrix, which could bias the analysis [13]. We followed an EWAS strategy to reduce the number of $\mathrm{CpGs}$ to analyze from the methylation data, selecting the 20,000 CpGs with the lowest $p$-value in the association with CVD. As a sensitivity analysis, we also selected the 20,000 CpGs with the highest variability measured by the standard deviation (recommended by MOFA authors). Data, model and training options were left as default, but the "convergence_mode" train argument was set to "slow" and the "num_factors" to 30 .

We determined the variance explained per factor in both omics, and the total variance explained by each omic. As a quality control, we estimated the correlation between factors to check whether they captured unique sources of variation.
MOFA is a completely unsupervised machine-learning method, and the covariates and the presence of CVD were not used for model training. The relationship between the presence of CVD, the covariates and the MOFA factors was analyzed a posteriori.

\section{Statistical analysis}

First, the association between the identified MOFA factors and CVD incidence was assessed using Cox proportional hazards regression models using survival (v3.1-12) [57] and Hmisc (v4.4-0) [58] R packages. We defined three models for each MOFA factor: non-adjusted, adjusted for sex and age and additionally adjusted for total cholesterol, HDL-C, SBP, DBP, glucose and smoking. Cell-type counts and one surrogate variable were used as covariates in the three models. We also tested the interaction between the MOFA factors and sex on CVD risk.

Second, we assessed the potential added predictive value of including the CVD-associated MOFA factors in the Framingham risk function by estimating the improvement in discrimination (Harrell's c statistic), applying the rcorr.cens function of the Hmisc R package, and the net reclassification improvement (NRI), using the nricens $\mathrm{R}$ package (v1.6) [59]. We defined three risk categories (low, intermediate and high), applying cutoffs according to National Cholesterol Education Panel (NCEP) guidelines for 10-year risk [60]: [0-10]\%, [10-20)\%, $\geq 20 \%$, respectively). The expected number of events at 5 years in each risk category (thus, $[0-5] \%,[5-10] \%, \geq 10 \%$ ) were calculated using Kaplan-Meier estimates. Moreover, we analyzed the NRI in the group of individuals with intermediate CVD risk-i.e., the clinical NRI-and corrected the bias in NRI estimation in this group [61].

\section{Biological pathways of the CVD-related MOFA factors}

Each MOFA factor is defined by several features of the integrated omics (either CpGs or expressed genes). Features with score values close to zero are not related to the factor, whereas features with large absolute values have a strong association with it. The sign of the weight indicates the direction of the association. We identified the features with the highest scores defining the factors related to CVD and, using the corrplot R package (v0.84) [62], estimated the correlation between all the features included in one factor to identify those that captured unique sources of variation. The top $30 \mathrm{CpGs}$ within each factor were checked in the EWAS catalog [63], and we annotated the expressed genes using the Affymetrix HuEx-1_0-st-v2 annotation file. Finally, we assessed the association between each of the top 30 features of each factor and CVD risk using Cox regression models. 


\section{Sensitivity analysis and independent replication of the MOFA factors and the top CpG features related to CVD}

As a sensitivity analysis, we examined the association between the identified MOFA factors and CHD, to assess the consistency of the effect sizes of the associations between MOFA factors and CVD, and those with CHD.

Two approaches were used to replicate the main DNA methylation markers identified as relating to CVD in an independent EWAS from the REGICOR study [64]. This study included 208 consecutive myocardial infarction cases (104 women, overrepresented in the study) and 208 age- and sex-matched controls. DNA methylation was assessed with the Illumina HumanMethylationEPIC array, and data quality control was very similar to that performed in the FOS population [64]. Additional information can be found in Additional file 3. First, we ran a new and similar analysis in the replication cohort REGICOR, using the 40,000 CpGs more significantly associated with myocardial infarction in the REGICOR study (those with the lowest $p$-value in the EWAS). Thus, we identified latent factors using the MOFA2 $\mathrm{R}$ package and assessed their association with myocardial infarction. Then, we assessed whether the MOFA factors related to CVD (in FOS) and myocardial infarction (in REGICOR) pointed to similar significant biological pathways. Second, we identified the top $30 \mathrm{CpGs}$ that defined the MOFA factors related to CVD in the FOS and assessed for their association with myocardial infarction in the REGICOR study.

\section{Abbreviations \\ CHD: Coronary heart disease; CVD: Cardiovascular diseases; dbGAP: Database of genotypes and phenotypes; DBP: Diastolic blood pressure; EWAS: Epige- nome-wide association studies; FDR: False discovery rate; FOS: Framingham offspring study; HDL-C: High-density lipoprotein cholesterol; MOFA: Multi- omics factor analysis; REGICOR: REgistre Glroní del COR; SBP: Systolic blood pressure.}

\section{Supplementary Information}

The online version contains supplementary material available at https://doi. org/10.1186/s13148-021-01064-y.

Additional file 1: Supplementary tables.

Additional file 2: Supplementary figures.

Additional file 3: Supplementary material and methods.
(GP-M, AF-S, RE) or revising it critically for important intellectual content (IS, LN). Final approval of the version to be published (GP-M, IS, LN, AF-S, RE). Agreement to be accountable for all aspects of the work in ensuring that questions related to the accuracy or integrity of any part of the work are appropriately investigated and resolved (GP-M, IS, LN, AF-S, RE). All authors read and approved the final manuscript.

\section{Funding}

This project was funded by the Carlos III Health Institute-European Regional Development Fund (FIS PI18/00017, CIBERCV, CIBERESP), PERIS from Agència de Gestió d'Ajuts Universitaris i de Recerca (SLT002/16/00088) and the Government of Catalonia through the Agency for Management of University and Research Grants (2017SGR946). Fernández-Sanlés was funded by the Spanish Ministry of Economy and Competitiveness (BES-2014-069718).

\section{Availability of data and materials}

The datasets analyzed during the current study are available in the dbGAP repository: https://www.ncbi.n/m.nih.gov/projects/gap/cgi-bin/study.cgi? study_id=phs000007.v31.p12. Specifically, the dbGaP Study Accession codes were phs000724.v7.p11 and phs000363.v17.p11, for the DNA methylation and gene expression data, respectively. The code underlying this article is available at https://github.com/gpalou4/TFM. Supplementary information: Supplementary material is available at https://github.com/gpalou4/TFM/tree/master/ manuscript/supp_material

\section{Declarations}

\section{Ethics approval and consent to participate}

FOS data were obtained through the database of Genotypes and Phenotypes (dbGAP, http://dbgap.ncbi.nlm.nih.gov; project number \#9047). The study was approved by the Parc Salut Mar ethics committee (2015/6199/1; 2018/7855/I) and meets the principles expressed in the Declaration of Helsinki and the relevant European legislation.

\section{Consent for publication}

Not applicable.

\section{Competing interests}

The authors declare that they have no competing interests. The Framingham Heart Study (FHS) is conducted and supported by the US National Heart, Lung and Blood Institute (NHLBI) in collaboration with Boston University (Contract No. N01-HC-25195 and HHSN268201500001I). This manuscript was not prepared in collaboration with investigators of the FHS, has not been reviewed and/or approved by the FHS and does not necessarily reflect the opinions or views of the FHS investigators or the NHLBI.

\section{Author details}

${ }^{1}$ Cardiovascular Epidemiology and Genetics Research Group, Hospital del Mar Medical Research Institute (IMIM), Dr Aiguader 88, 08003 Barcelona, Spain. ${ }^{2}$ Pompeu Fabra University (UPF), Barcelona, Spain. ${ }^{3}$ Institute for Research in Biomedicine (IRB Barcelona), The Barcelona Institute of Science and Technology, Barcelona, Spain. ${ }^{4}$ CIBER Epidemiology and Public Health (CIBERESP), Barcelona, Spain. ${ }^{5}$ MARGenomics, Hospital del Mar Medical Research Institute (IMIM), Barcelona, Spain. ${ }^{6}$ MRC Integrative Epidemiology Unit at the University of Bristol, Bristol, UK. ${ }^{7}$ CIBER Cardiovascular Diseases (CIBERCV), Barcelona, Spain. ${ }^{8}$ Medicine Department, Faculty of Medicine, University of Vic-Central University of Catalonia (UVic-UCC), Vic, Spain.

Received: 8 January 2021 Accepted: 29 March 2021

Published online: 09 April 2021

\section{References}

1. Roth GA. Global, regional, and national age-sex-specific mortality for 282 causes of death in 195 countries and territories, 1980-2017: a systematic analysis for the Global Burden of Disease Study 2017. Lancet. 2018;392:1736-88.

2. James SL. Global, regional, and national incidence, prevalence, and years lived with disability for 354 Diseases and Injuries for 195 countries and
Substantial contributions to the conception or design of the work (AF-S, RE); or the acquisition (RE), EWAS analysis (AF-S), integration analysis (GP-M) or interpretation of data for the work (GP-M, AF-S, IS, LN, RE). Drafting the work
We thank Ricard Argelaguet for his comments and help with the MOFA analyses and Elaine M. Lilly, Ph.D., for her critical reading and revision of the English text.

\section{Authors' contributions}


territories, 1990-2017: a systematic analysis for the Global Burden of Disease Study 2017. Lancet. 2018;392:1789-858.

3. Piepoli MF, Abreu A, Albus C, Ambrosetti M, Brotons C, Catapano AL, et al. Update on cardiovascular prevention in clinical practice: a position paper of the European Association of Preventive Cardiology of the European Society of Cardiology. Eur J Prev Cardiol. 2020;27:181-205.

4. Andersson C, Johnson AD, Benjamin EJ, Levy D, Vasan RS. 70-year legacy of the Framingham Heart Study. Nat Rev Cardiol. 2019;16:687-98.

5. Sampson UK, Fazio S, Linton MF. Residual cardiovascular risk despite optimal LDL cholesterol reduction with statins: the evidence, etiology, and therapeutic challenges. Curr Atheroscler Rep. 2012;14:1-10.

6. Erdmann J, Kessler T, Munoz Venegas L, Schunkert H. A decade of genome-wide association studies for coronary artery disease: the challenges ahead. Cardiovasc Res. 2018;114:1241-57.

7. Fernández-Sanlés A, Sayols-Baixeras S, Subirana I, Degano IR, Elosua R. Association between DNA methylation and coronary heart disease or other atherosclerotic events: a systematic review. Atherosclerosis. 2017;263:325-33.

8. Agha G. Blood leukocyte DNA methylation predicts risk of future myocardial infarction and coronary heart disease. Circulation. 2019;140:645-57.

9. Ward-Caviness CK, Agha G, Chen BH, Pfeiffer L, Wilson R, Wolf P, et al. Analysis of repeated leukocyte DNA methylation assessments reveals persistent epigenetic alterations after an incident myocardial infarction. Clin Epigenetics. 2018;10:161.

10. Nurnberg ST, Guerraty MA, Wirka RC, Rao HS, Pjanic M, Norton S, et al. Genomic profiling of human vascular cells identifies TWIST1 as a causal gene for common vascular diseases. PLOS Genet. 2020;16:e1008538.

11. Joshi A, Rienks M, Theofilatos K, Mayr M. Systems biology in cardiovascular disease: a multiomics approach. Nat Rev Cardiol. 2020; Dec 18. https:// doi.org/10.1038/s41569-020-00477-1. Online ahead of print.

12. Zampieri G, Vijayakumar S, Yaneske E, Angione C. Machine and deep learning meet genome-scale metabolic modeling. PLOS Comput Biol. 2019;15:e1007084.

13. Argelaguet R, Velten B, Arnol D, Dietrich S, Zenz T, Marioni JC, et al. Multi-omics factor analysis - a framework for unsupervised integration of multi-omics data sets. Mol Syst Biol. 2018;14:1-13.

14. Argelaguet R, Arnol D, Bredikhin D, Deloro Y, Velten B, Marioni JC, et al. MOFA+: a probabilistic framework for comprehensive integration of structured single-cell data. bioRxiv. 2019;837104.

15. Pierre-Jean M, Deleuze J-F, Le Floch E, Mauger F. Clustering and variable selection evaluation of 13 unsupervised methods for multi-omics data integration. Brief Bioinform. 2019;bbz138.

16. McCabe SD, Lin D-Y, Love MI. Consistency and overfitting of multi-omics methods on experimental data. Brief Bioinform. 2019;bbz070.

17. Lappalainen T, Greally JM. Associating cellular epigenetic models with human phenotypes. Nat Rev Genet. 2017;18:441-51.

18. Kennel PJ, Liao X, Saha A, Ji R, Zhang X, Castillero E, et al. Impairment of myocardial glutamine homeostasis induced by suppression of the amino acid carrier SLC1A5 in failing myocardium. Circ Hear Fail. 2019;12:e006336.

19. Prompunt E, Sanit J, Barrère-Lemaire S, Nargeot J, Noordali H, Madhani $M$, et al. The cardioprotective effects of secretory leukocyte protease inhibitor against myocardial ischemia/reperfusion injury. Exp Ther Med. 2018:15:5231-42.

20. Peng $P$, Wang $L$, Yang $X$, Huang $X, B a Y$, Chen $X$, et al. A preliminary study of the relationship between promoter methylation of the ABCG1, GALNT2 and HMGCR genes and coronary heart disease. PLOS ONE. 2014;9:e102265.

21. Poulet M, Sirois J, Boyé K, Uetani N, Hardy S, Daubon T, et al. PRL-2 phosphatase is required for vascular morphogenesis and angiogenic signaling. Commun Biol. 2020;3:603.

22. Shang J, Gao Z-Y, Zhang L-Y, Wang C-Y. Over-expression of JAZF1 promotes cardiac microvascular endothelial cell proliferation and angiogenesis via activation of the Akt signaling pathway in rats with myocardial ischemia-reperfusion. Cell Cycle. 2019:18:1619-34.

23. der Pim VH, Niek V. Identification of 64 novel genetic loci provides an expanded view on the genetic architecture of coronary artery disease. Circ Res. 2018;122:433-43.

24. Hata J, Mukai N, Nagata M, Ohara T, Yoshida D, Kishimoto H, et al. Serum angiopoietin-like protein 2 is a novel risk factor for cardiovascular disease in the community: the Hisayama study. Arterioscler Thromb Vasc Biol. 2016:36:1686-91.
25. Lotta LA Wittemans LBL, Zuber V Stewart ID, Sharp SJ, Luan J, et al. Association of genetic variants related to gluteofemoral vs abdominal fat distribution with type 2 diabetes, coronary disease, and cardiovascular risk factors. JAMA. 2018;320:2553.

26. Dogan MV, Grumbach IM, Michaelson JJ, Philibert RA. Integrated genetic and epigenetic prediction of coronary heart disease in the Framingham Heart Study. PLoS ONE. 2018:13:1-18.

27. Gross AM, Jaeger PA, Kreisberg JF, Licon K, Jepsen KL, Khosroheidari M, et al. Methylome-wide analysis of chronic HIV infection reveals five-year increase in biological age and epigenetic targeting of HLA. Mol Cell. 2016:62:157-68.

28. Ahola-Olli AV, Würtz P, Havulinna AS, Aalto K, Pitkänen N, Lehtimäki T, et al Genome-wide association study identifies 27 loci influencing concentrations of circulating cytokines and growth factors. Am J Hum Genet. 2017;100:40-50.

29. Han X, Ong J-S, An J, Hewitt AW, Gharahkhani P, MacGregor S. Using Mendelian randomization to evaluate the causal relationship between serum C-reactive protein levels and age-related macular degeneration. Eur J Epidemiol. 2020;35:139-46.

30. Astle WJ, Elding H, Jiang T, Allen D, Ruklisa D, Mann AL, et al. The allelic landscape of human blood cell trait variation and links to common complex disease. Cell. 2016;167:1415-29.

31. Ding $X, H e Y$, Hao $Q$, Chen $S$, Yang $M$, Leng $S X$, et al. The association of single nucleotide polymorphism rs189037C > T in ATM gene with coronary artery disease in Chinese Han populations. Medicine (Baltimore). 2018;97:e9747.

32. Nakatochi M, Ichihara S, Yamamoto K, Naruse K, Yokota S, Asano H, et al. Epigenome-wide association of myocardial infarction with DNA methylation sites at loci related to cardiovascular disease. Clin Epigenetics. 2017;9:54.

33. Xi X, Zhang J, Wang J, Chen Y, Zhang W, Zhang X, et al. SGK1 mediates hypoxic pulmonary hypertension through promoting macrophage infiltration and activation. Anal Cell Pathol. 2019;2019:1-10.

34. Das S, Aiba T, Rosenberg M, Hessler K, Xiao C, Quintero PA, et al. Pathological role of serum- and glucocorticoid-regulated kinase 1 in adverse ventricular remodeling. Circulation. 2012;126:2208-19.

35. Liu C, Marioni RE, Hedman ÅK, Pfeiffer L, Tsai P-C, Reynolds LM, et al. A DNA methylation biomarker of alcohol consumption. Mol Psychiatry. 2018;23:422-33.

36. Wahl S, Drong A, Lehne B, Loh M, Scott WR, Kunze S, et al. Epigenomewide association study of body mass index, and the adverse outcomes of adiposity. Nature. 2017:541:81-6.

37. Joehanes R, Just AC, Marioni RE, Pilling LC, Reynolds LM, Mandaviya PR, et al. Epigenetic signatures of cigarette smoking. Circ Cardiovasc Genet. 2016;9:436-47.

38. Kessler T, Vilne B, Schunkert $H$. The impact of genome-wide association studies on the pathophysiology and therapy of cardiovascular disease. EMBO Mol Med. 2016;8:688-701.

39. Buniello A, MacArthur JAL, Cerezo M, Harris LW, Hayhurst J, Malangone C, et al. The NHGRI-EBI GWAS catalog of published genome-wide association studies, targeted arrays and summary statistics 2019. Nucleic Acids Res. 2019;47:D1005-12.

40. Davis W, Tew KD. ATP-binding cassette transporter-2 (ABCA2) as a therapeutic target. Biochem Pharmacol. 2018;151:188-200.

41. Joehanes $R$, Ying $S$, Huan $T$, Johnson AD, Raghavachari N, Wang R, et al. Gene expression signatures of coronary heart disease. Arterioscler Thromb Vasc Biol. 2013;33:1418-26.

42. Sillanpää MJ. Overview of techniques to account for confounding due to population stratification and cryptic relatedness in genomic data association analyses. Heredity. 2011;106:511-9.

43. Barfield RT, Almli LM, Kilaru V, Smith AK, Mercer KB, Duncan R, et al. Accounting for population stratification in DNA methylation studies. Genet Epidemiol. 2014;38:231-41.

44. Buettner F, Theis FJ. A novel approach for resolving differences in singlecell gene expression patterns from zygote to blastocyst. Bioinformatics. 2012;28:i626-32.

45. Sayols-Baixeras S, Subirana I, Lluis-Ganella C, Civeira F, Roquer J, Do A, et al. Identification and validation of seven new loci showing differential DNA methylation related to serum lipid profile: an epigenome-wide approach. The REGICOR study Hum Mol Genet. 2016;25:4556-65. 
46. Bibikova M, Barnes B, Tsan C, Ho V, Klotzle B, Le JM, et al. High density DNA methylation array with single $\mathrm{CpG}$ site resolution. Genomics, 2011;98:288-95.

47. Sandoval J, Heyn HA, Moran S, Serra-Musach J, Pujana MA, Bibikova M, et al. Validation of a DNA methylation microarray for $450,000 \mathrm{CpG}$ sites in the human genome. Epigenetics. 2011;6:692-702.

48. Chen YA, Lemire M, Choufani S, Butcher DT, Grafodatskaya D, Zanke BW, et al. Discovery of cross-reactive probes and polymorphic CpGs in the Illumina Infinium HumanMethylation450 microarray. Epigenetics. 2013;8:203-9.

49. Zhou W, Laird PW, Shen H. Comprehensive characterization, annotation and innovative use of Infinium DNA methylation BeadChip probes. Nuc Acids Res. 2017:45:e22.

50. Pidsley RY, Wong CC, Volta M, Lunnon K, Mill J, Schalkwyk LC. A datadriven approach to preprocessing Illumina $450 \mathrm{~K}$ methylation array data. BMC Genomics. 2013;14:293.

51. Aryee MJ, Jaffe AE, Corrada-Bravo H, Ladd-Acosta C, Feinberg AP, Hansen $K D$, et al. Minfi: A flexible and comprehensive bioconductor package for the analysis of Infinium DNA methylation microarrays. Bioinformatics. 2014:30:1363-9.

52. Johnson WE, Li C, Rabinovic A. Adjusting batch effects in microarray expression data using empirical Bayes methods. Biostatistics. 2007;8:118-27.

53. Jaffe AE. FlowSorted.Blood.450k: Illumina HumanMethylation data on sorted blood cell populations. R package. 2019

54. Leek JT, Johnson WE, Parker HS, Fertig EJ, Jaffe AE, Zhang Y, et al. sva: Surrogate Variable Analysis. R package. 2019.

55. Carvalho BS, Irizarry RA. A framework for oligonucleotide microarray preprocessing. Bioinformatics. 2010;26:2363-7.
56. Irizarry RA, Hobbs B, Collin F, Beazer-Barclay YD, Antonellis KJ, Scherf U, et al. Exploration, normalization, and summaries of high density oligonucleotide array probe level data. Sel Work Terry Speed. 2012;601-16.

57. Therneau T. A Package for Survival Analysis in R. R package [Internet]. 2020. Available from: https://cran.r-project.org/package=survival

58. Harrell FE. Hmisc: Harrell Miscellaneous. R package [Internet]. 2020. Available from: https://cran.r-project.org/web/packages/Hmisc/

59. Pencina MJ, D'Agostino RB, Steyerberg EW. Extensions of net reclassification improvement calculations to measure usefulness of new biomarkers. Stat Med. 2011;30:11-21.

60. NCEP. Third Report of the National Cholesterol Education Program (NCEP) Expert Panel on Detection, Evaluation, and Treatment of High Blood Cholesterol in Adults (Adult Treatment Panel III) Final Report. Circulation. 2002;106:3143.

61. Paynter NP, Cook NR. A bias-corrected net reclassification improvement for clinical subgroups. Med Decis Mak. 2013;33:154-62.

62. Wei T, Simko V. "corrplot": Visualization of a Correlation Matrix. R package [Internet]. 2017. Available from: https://cran.r-project.org/web/packages/ corrplot/

63. Staley J. EWAS catalog [Internet]. 2020. Available from: http://www.ewasc atalog.org

64. Fernández-Sanlés A, Sayols-Baixeras S, Subirana I, Sentí M, PérezFernández S, Castro de Moura M, et al. DNA methylation biomarkers of myocardial infarction and cardiovascular disease. bioRxiv. 2019;3:707315.

\section{Publisher's Note}

Springer Nature remains neutral with regard to jurisdictional claims in published maps and institutional affiliations.
Ready to submit your research? Choose BMC and benefit from:

- fast, convenient online submission

- thorough peer review by experienced researchers in your field

- rapid publication on acceptance

- support for research data, including large and complex data types

- gold Open Access which fosters wider collaboration and increased citations

- maximum visibility for your research: over 100M website views per year

At BMC, research is always in progress.

Learn more biomedcentral.com/submissions 